\title{
Ways of working at the interface between primary and specialist mental healthcare
}

\author{
Linda Gask and Tarun Khanna
}

\section{Summary}

Ways of working at the interface between primary and specialist care are considered with discussion of the limits of available evidence and the potential for a new role for psychiatrists in providing supervision and consultation in novel models of care.

\section{Declaration of interest}

L.G. has received payment for speaking from Lilly, wyeth, Lundbeck and Pfizer, and research funding from Servier and Pfizer
Linda Gask (pictured) is at the National School for Primary Care Research at the University of Manchester and the Salford Primary Care NHS Trust. Tarun Khanna is at the University of Manchester and Greater Manchester West Mental Health NHS Trust.

There is considerable international policy interest in cost-effective ways of working at the interface between primary and specialist care across medical specialties. ${ }^{1}$ Although high-quality evidence to inform the development of services is still in short supply, some conclusions can be drawn about the current state of the field, including the likely impact of novel ways of working on the role of psychiatrists.

\section{Models of interface working}

A range of models for working at the primary-specialist interface are described in the international research literature (online Appendix DS1). Community mental health teams (CMHTs) and attached mental health professional models have been universally implemented across the UK, unlike consultation-liaison and collaborative care. Two further models of 'stepped care' and 'matched' care (operated by triage or 'gateway' workers), may be combined with the above, to determine how decisions are made about access at the interface.

\section{Community mental health teams}

Community mental health teams (multidisciplinary teams) in the UK have responded to national policy imperatives to focus care on and improve multidisciplinary treatment of severe and enduring mental illness, for which psychotic symptoms have served as a proxy measure. Within teams psychiatrists have generally managed higher case-loads, including a higher proportion of service users with a diagnosis of psychosis. Tensions arise from the need to provide a first point of assessment for referrals from primary care, and the ongoing provision of care for people with longer-term mental illness.

The response of CMHTs to the problem of managing demand where resources are limited, particularly in inner-city areas, has been to limit access to care, utilising 'gate-keeping' and 'triage' models to more tightly (and bureaucratically) manage the interface. Where access is gained to the service provided by the CMHT, outcomes seem to be at least equivalent to traditional out-patient care, with improved outcomes when teams have smaller case-loads, operate assertive follow-up and are trained to deliver psychosocial interventions. ${ }^{2}$ However, outcomes are less satisfactory for those who fail to meet criteria for severe and enduring mental illness or present with personal and social problems for which the CMHT has limited capacity to intervene.

Restriction of access had led to widespread dissatisfaction in primary care, which values the opinion of the psychiatrist in managing complex problems such as treatment-resistant depression. Such a referral may not be accepted by team managers. Within the teams there has also been the effect of restriction of the professional role of workers (especially psychiatrists) to the assessment and management of psychosis. In the UK, CMHTs have experimented with looser collaboration through 'link working' in which members of the team liaise with particular primary care providers, and with changes to their ways of working to improve efficiency by introduction of 'one-off' assessments and/or initial telephone assessment of referrals.

\section{Attached professionals}

The therapeutic work of attached professionals is highly congruent with what patients and general practitioners (GPs) want; is strongly supported by current health policy; ${ }^{3}$ has a high level of accountability in the specific professions involved in delivery (psychologists, counsellors) and the confidence of those who request it (GPs). However, these professionals still work in a way that is essentially disengaged from primary care rather than integrated with it. Access is often problematic with long waiting lists, and can only be improved if there is a large enough cohort of suitably trained and supervised therapists available to meet demand, and there is a very limited role for psychiatrists in the current UK policy to focus on psychological therapy. ${ }^{3}$ The effects of attached mental health professionals on patient outcome are variable, depending partly on the type of intervention provided, and are primarily for the short term, tailing off over time. Notably, care provided by community mental health nurses for common mental disorders has not been shown to demonstrably improve outcomes over routine GP care. ${ }^{4}$

\section{Consultation-liaison}

In consultation-liaison there are regular conversations between specialists and the primary care team, which may vary from brief corridor conversations to more formal meetings. Where there is wider involvement of a CMHT in these meetings, the meetings tend to focus on service users already known to the mental health 
service rather than on new cases. Consultation-liaison services have been offered in combination with brief therapeutic contact with attached mental health specialists in several countries including the USA, Canada and Italy. In rural areas, consultation-liaison has been provided successfully via teleconferencing.

Consultation-liaison differs from the other models in that it challenges the way that professionals relate to each other across organisational boundaries. However, despite early enthusiasm for this model, it seems to be of limited interest to both GPs and psychiatrists in the current context of secondary-care services being more focused on severe mental illness; and there is no evidence that it has an impact on outcomes. ${ }^{5}$

\section{Collaborative care}

Collaborative care has itself been variously defined to mean everything from collaboration between services and 'shared care' to the more highly structured definition now internationally used (online Appendix DS1). Collaborative care further introduces novel working practices not just across organisational boundaries but between patients and professionals. Sessions may be highly structured and protocol-driven, with extensive use of the telephone. New workers, 'case managers', systematically follow-up individuals under regular supervision and usually provide both brief psychological therapy and medication management. There is a growing body of international evidence that collaborative care improves outcome with depression, ${ }^{6}$ with more limited evidence for anxiety; however, the National Institute for Health and Clinical Excellence guidelines currently support collaborative care only for depression with chronic physical illness. ${ }^{7}$ A collaborative-care model for people with longer-term mental health problems discharged back to primary care in Australia showed no deterioration in outcome compared with those who remained in a mental health service. ${ }^{8}$

Implementation and workability in practice is also highly dependent on having sufficient well-trained and supervised case managers to manage the majority of the workload that does not require specialist input. A combination of stepped and collaborative care (increasing intensity of care by 'stepping up' only if required) may both increase access and manage demand, but will require probably increased investment at least in the short term. Indeed, although there is some suggestion of longer-term savings in the implementation of collaborative care for depression in the US setting of managed care, ${ }^{7}$ this may be true only for older people or those with comorbidities. It may not translate to a setting where there is less medical input into the usual care of people with common mental health problems.

\section{Accessing care}

Matched-care models (usually operated by triage and gateway workers in the UK) may result in multiple assessments for the patient in gaining access to a place where treatment is provided. There has been very little formal evaluation of the impact of the gateway workers who provide triage in the UK and, internationally, research into triage is similarly limited, although nurses who provide telephone triage may feel insufficiently supervised and supported.

In a system that operates a model of stepped care, individuals will be 'stepped up' or 'stepped down' the hierarchy of treatment according to how they progress. There is limited evidence that stepped care has similar outcomes to matched care. ${ }^{9}$ Nevertheless, there is patient dissatisfaction related to unmet expectations of care when minimal interventions are offered initially in stepped care. General practitioners in the UK appear to dislike the movement towards a 'single point of access' to services where individuals are triaged and matched to the appropriate level of care because it not only seems like 'referring into the void' ${ }^{10}$ but impedes the establishment of professional relationships and transfer of knowledge (and mental health professionals in this study seemed unaware of GPs' negative views).

\section{Implications for the role of the psychiatrist}

Newer models of working at the interface such as consultationliaison, collaborative care and novel approaches to access such as stepped care potentially place the psychiatrist in a key supervisory role, with availability to consult with a range of professionals and develop care plans for individuals with more complex problems. In mental healthcare, and particularly where novel ways of working have been established, initial assessment tasks are increasingly carried out by nursing staff and case managers. In order to work successfully with primary care, psychiatrists need to acquire skills in the brief assessment and management of a wide range of common mental disorders and provide supervision to and consultation with others providing this care. This is difficult if opportunities for acquisition of these skills are not available to trainees. Of particular importance is the ability to communicate effectively with primary care and to provide support for front-line staff, including mental health workers and GPs.

Although the research into collaborative and stepped care has clearly designated such a role for mental health professionals, psychiatry is generally yet to consider how to develop this way of working. There is a potential opportunity for 'primary care psychiatrists' to play a key part in developing the interface with primary care. However, it will be necessary to provide training for this role.

Linda Gask, PhD, FRCPsych, National School for Primary Care Research, University of Manchester and Salford Primary Care NHS Trust; Tarun Khanna, BSC (Med SCi), MBChB, School of Community-Based Medicine, University of Manchester and Greater Manchester West Mental Health NHS Trust, UK.

Correspondence: Linda Gask, PhD, FRCPsych, National School for Primary Care Research, 5th Floor Williamson Building, University of Manchester, Oxford Road, Manchester M13 9PL, UK. Email: linda.gask@manchester.ac.uk

First received 21 May 2010, final revision 24 Aug 2010, accepted 9 Sep 2010

\section{References}

1 World Health Organization. Integrating Mental Health into Primary Care: A Global Perspective. WHO, 2008.

2 Burns T. Influencing community mental health team practice to improve care outcomes. In Choosing Methods in Mental Health Research: Mental Health Research from Theory to Practice (eds M Slade \& S Priebe): 138-46. Taylor \& Francis, 2006.

3 Layard R. The case for psychological treatment centres. BMJ 2006; 332 1030-2.

4 Kendrick T, Simons L, Mynors-Wallis L, Gray A, Lathlean J, Pickering R, et al. Cost-effectiveness of referral for generic care or problem-solving treatment from community mental health nurses, compared with usual general practitioner care for common mental disorders. Randomised controlled trial. Br J Psychiatry 2006; 189: 50-9.

5 Cape J, Whittington C, Bower P. What is the role of consultation-liaison psychiatry in the management of depression in primary care? A systematic review and meta-analysis. Gen Hosp Psychiatry 2010; 32: 246-54.

6 Gilbody S, Bower P, Fletcher J, Richards D, Sutton AJ. Collaborative care for depression: a cumulative meta-analysis and review of longer-term outcomes. Arch Intern Med 2006; 166: 2314-21. 
7 National Institute for Health and Clinical Excellence. Depression in Adults with a Chronic Physical Health Problem. NICE, 2009 (http:// guidance.nice.org.uk/CG91/Guidance).

8 Meadows GN, Harvey CA, Joubert L, Barton D, Bedi G. Best practices: the consultation-liaison in primary-care psychiatry program: a structured approach to long-term collaboration. Psychiatr Serv 2007; 58: 1036-8.
9 van Straten A, Tiemens B, Hakkaart L, Nolen WA, Donker MC. Stepped care vs. matched care for mood and anxiety disorders: a randomized trial in routine practice. Acta Psychiatr Scand 2006; 113: 468-76.

10 Raine R, Carter S, Sensky T, Black N. 'Referral into a void': opinions of general practitioners and others on single point of access to mental health care. J R Soc Med 2005; 98: 153-7.

\section{psychiatry in pictures}

\section{Ruddy Bob}

Marilyn George (2008)

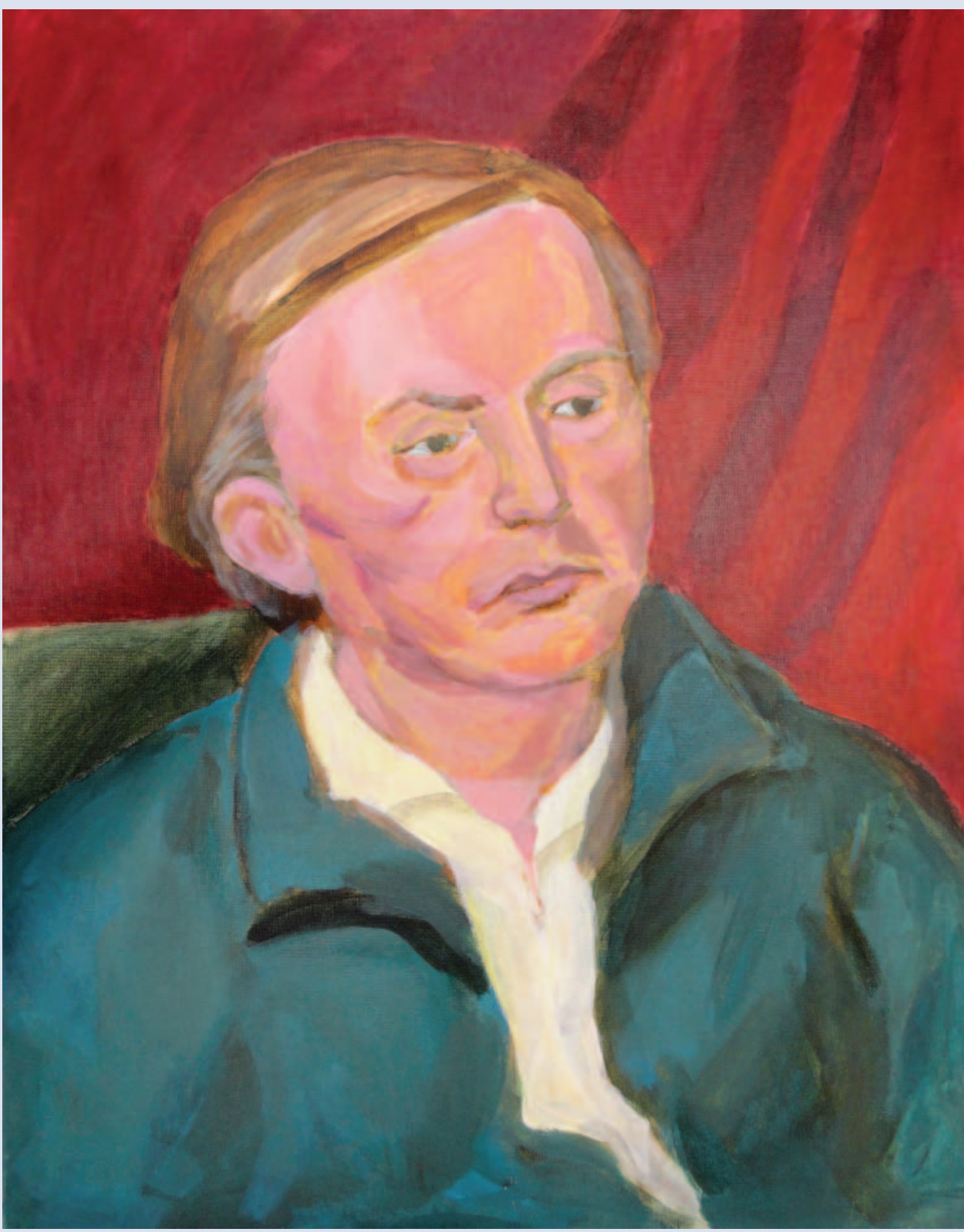

The painting was done in acrylic on canvas. The sitter's name is Bob - he was a model at the portrait class attended by the artist Marilyn George. The artist enjoyed a conversation with him about 60s pop groups; he enjoyed his youth in the 60s and the artist is a big fan of 60 s music.

The artist suffers from a severe mental illness of the schizophrenic spectrum. She has always enjoyed painting and finds herself inspired a lot by portrait painters of the past, particularly John singer Sargent whom she would like to emulate. Although unable to hold down a full-time job, the artist is quite ambitious about her painting and wants to produce work of very high quality. She finds it important to have goals in her life and she finds painting therapeutic.

The British Journal of Psychiatry (2011) 198, 5. doi: 10.1192/bjp.198.1.5 